\title{
Attenuation Coefficients for Propagation at Very Low Frequencies (VLF) During a Sudden Ionospheric Dis- turbance (SID) ${ }^{1}$
}

\author{
E. T. Pierce \\ Contribution from Stanford Research Institute. Menlo Park, Calif.
}

(Received May 11, 1961)

\begin{abstract}
Attenuation coefficients for propagation under SID conditions are deduced, from records of atmospheric noise, for the frequen y range of 3.5 to 50 kilocycles per second. These are compared with the values under normal daytime circumstances. It is shown that the advent of SID implies little change in attenuation between about 12 and 20 kilocycles per second; above this range there is a markedly decreased attenuation, while below 12 kilocycles per second there is an even more pronounced increase in the attenuation coefficients. The principle of an improved recorder of the changes in atmospheric noise associated with SID conditions is outlined; this recorder would discriminate between source effects independent of the SID and propagation influences solely attributable to the SID.
\end{abstract}

\section{Introduction}

The behavior, during propagation under normal daytime conditions, of radio waves of very low frequency (VLF), is now well established. It is known that the propagation is within the waveguide formed by the earth and the lower edge of the ionospheric $D$ layer. Attenuation coefficients in the VLF range (3 to $30 \mathrm{kc} / \mathrm{s}$ ) have been deduced using the mode theory [Wait, 1957], and have also been derived experimentally from observations of atmospherics [Chapman and Macario, 1956] and of ongwave transmitters [Watt and Plush, 1959]. The agreement between experimental and theoretical results has been good, and the increasing recgnition of such refinements as the influence of ground conductivity, the effect of the earth's magnetic field, and so on, is likely to lead to still better accord.

One influence that considerably affects daytime VLF propagation is the sudden ionospheric disturbance (SID). It is now generally agreed that a SID is caused by an increased flux of X-rays emitted by the sun during a solar flare [Friedman, 1959]. These X-rays enhance the ionization in the $D$ layer. One aspect of this increased ionization is that an additional shallow bulge of electrons forms upon the underside of the normal $D$ region. The exact shape of the bulge depends upon the spectral distribution of the X-rays in the incident fux [Pierce and Swift, 1960]; in all cases, however, the depression of the $D$ layer will be greatest at the subsolar point, and there will be appreciable affects upon the $D$ region within a radius of at least $3,000 \mathrm{~km}$ from the subsolar point.

As regards propagation at VLF the influence of a SID may be represented as a narrowing of the waveguide and, possibly, a change of the effective con-

1 Paper presented at the 1961 Spring Meetingof of the URS T Washington, D.C. ductivity at the upper boundary of the guide. Consequences upon propagation may therefore be anticipated and these are indeed found. The main effects are sudden phase anomalies (SPA) in which a phase-change in the skywave from a manmade transmitter is detected, due to the depression of the $D$ reflecting layer; sudden anomalies in field strength (SFA) which result from the changed phase relationship between the skywave and the groundwave, again from an artificial transmitter; and sudden enhancements of atmospherics (SEA) in which an increase of the noise due to distant thunderstorms is observed (typically at a frequency of $27 \mathrm{kc} / \mathrm{s}$ ). SPA and SFA studies indicate that for a specific electron density an average SID lowers the $D$ layer by some $5 \mathrm{~km}$ [Bracewell and Straker, 1949].

Although, as already mentioned, VLF attenuation coefficients have been widely obtained for normal conditions, the same information does not appear to have been derived under SID circumstances. The essential raw material, namely the changes observed in field strengths, has however been available for some time. It is the purpose of this note to attempt to obtain some indication of the VLF attenuation coefficients, during a SID, from data upon atmospheries.

\section{Normal Daytime Attenuation Coefficients}

Figure 1 depicts the relationship between the attenuation coefficient and frequency during normal daytime propagation. Two curves are shown. The first curve was deduced by Chapman and Macario [1956] from observations of atmospherics. The second is that derived by Watt and Plush [1959], largely on the basis of observations of manmade transmitters, and is described as being an average for a mixed path. There are divergencies between the two curves but these are not entirely unexpected. Even if $D$ layer ionospheric conditions were uniform 


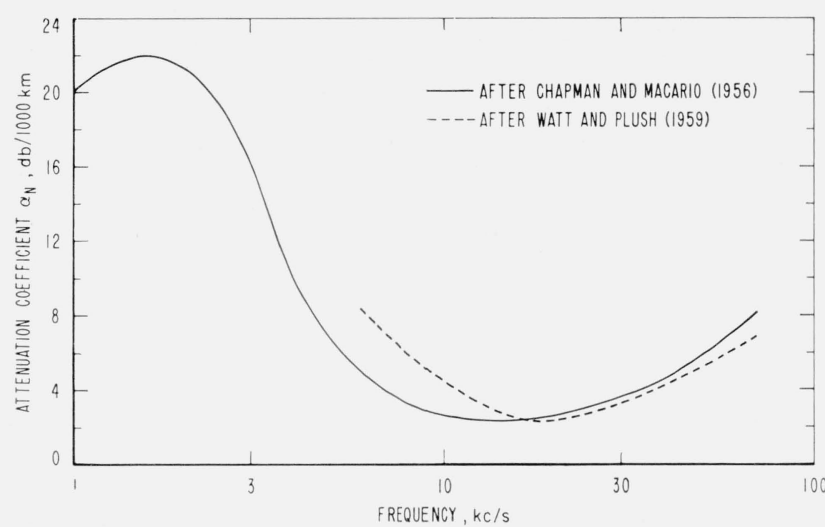

Figure 1. Attenuation coefficients for normal daytime conditions.

during daylight irrespective of solar altitude, such influences as ground conductivity and the direction of propagation with respect to the earth's magnetic field would still be important. These influences may lead to systematic deviations from "average" behavior. For instance, it is not unusual for the main sources of atmospherics received at a particular station to lie within a restricted geographical area; this implies that the attenuation coefficients, obtained from such observations of atmospherics, will be representative of a certain path, of its orientation with regard to the magnetic field and of the conductivity along its ground boundary, rather than of more general conditions.

\section{Basic Data and Its Analysis}

Wait [1958] has given the following expression for the vertical electric field $E$ (in millivolts/meter) at a great circle distance $d$ from a source radiating $P \mathrm{kw}$ :

$$
E \simeq \frac{300}{h}\left\{\frac{P \lambda}{a \sin (d / a)}\right\}^{\frac{1}{2}} \exp (-\alpha d)
$$

where $a$ is the radius of the earth, $h$ the height of the ionospheric reflecting layer in kilometers, $\lambda$ is the wavelength in kilometers, and $\alpha$ the attenuation factor. Equation (1) assumes that only a single mode is dominant, but this is a fair approximation up to $30 \mathrm{kc} / \mathrm{s}$ and for distances in the range 1,000 to $2,000 \mathrm{~km}$, and almost completely true for $d$ greater than $2,000 \mathrm{~km}$; thus (1) is valid in the study of distant atmospherics. Equation (1), however, is likely to be increasingly incorrect as the frequency rises above $30 \mathrm{kc} / \mathrm{s}$. Under normal conditions $(\operatorname{suffix} N)$ (1) may be written as

$$
E_{N}=\frac{300}{h_{N}}\left\{\frac{P \lambda}{a \sin (d / a)}\right\}^{\frac{1}{2}} \exp \left(-\alpha_{N} d\right) .
$$

If atmospheric noise is being received, then the advent of SID conditions (suffix $S$ ) will not cause $P$ or $d$ to change and

$$
E_{S}=\frac{300}{h_{S}}\left\{\frac{P \lambda}{a \sin (d / a)}\right\}^{\frac{1}{2}} \exp \left(-\alpha_{S} d\right) .
$$

Hence

$$
h_{N} E_{N} / h_{S} E_{S}=\exp \left(\alpha_{S}-\alpha_{N}\right) \mathrm{d},
$$

it being assumed that $E_{N}$ and $E_{S}$ are compared at the same frequency. Equation (4) may be converted into the form

$$
\left(\alpha_{S}-\alpha_{N}\right) d=2 \times 10^{4} \log _{10}\left(h_{N} E_{N} / h_{s} E_{s}\right),
$$

in which the units for $\alpha_{S}$ and $\alpha_{N}$ are now the familiar $\mathrm{db} / 1,000 \mathrm{~km}$. Thus if records of $E_{N}$ and $E_{S}$ are available together with estimates for $d, h_{N}$, and $h_{S},\left(\alpha_{S}-\alpha_{N}\right)$ can be deduced, and, knowing $\alpha_{N}, \alpha_{S}$ obtained.

Four sets of data in the published literature give the ratio $E_{N} / E_{S}$ at different frequencies and are therefore particularly suitable for the application of eq (5). These instances are in the papers by Gardner [1950], by Obayashi, Fujii, and Kidokoro [1959], and by Obayashi [1960]; this last paper contains two examples. Internal evidence within Gardner's paper suggests that $1,500 \mathrm{~km}$ is a good estimate for the distance of the noise sources; the reliability of this estimate is enhanced by the fact that Gardner worked in close collaboration with the Sferics Locating Network of the British Meteorological Office. It remains to determine $h_{N} / h_{S}$ before $\left(\alpha_{S}-\right.$ $\left.\alpha_{N}\right)$ can be calculated. $h_{N} / h_{S}$ is not far removed from unity and the errors in its estimation are considerably less than those implicit in assigning a value to $d$. $70 \mathrm{~km}$ is a commonly accepted value for $h_{N}$, while $h_{S}$ may be taken as $(65 \pm 5) \mathrm{km}$ [Bracewell and Straker, 1949] the extremes corresponding to the slightest and to the most intense SID. Thus $h_{N} / h_{S}=1.08$. Values of $E_{N} / E_{S}$ were obtained from Gardner's paper and, using eq (5), $\left(\alpha_{S}-\alpha_{N}\right)$ was calculated over the frequency range 3.5 to $50 \mathrm{kc} / \mathrm{s}$. The results are represented by curve (a) on figure 2 .

Similar information to that of Gardner is given in the much later paper by Obayashi and coworkers [1959]. Here the frequency range is restricted to

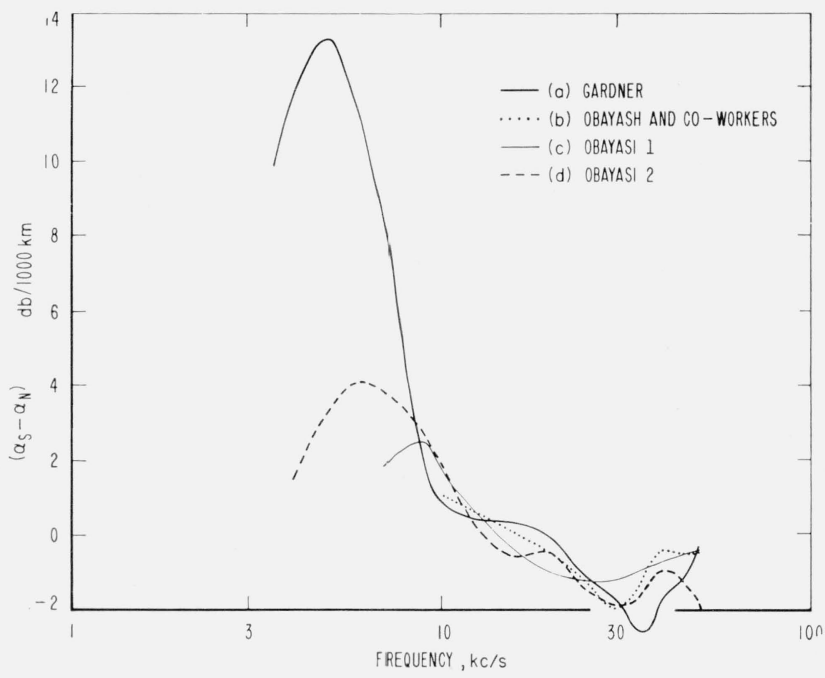

Figure 2. Difference between attenuation coefficients for normal daytime and for SID conditions. 
10 to $50 \mathrm{kc} / \mathrm{s}$. There is no direct evidence in the paper as to the distances of the sources of atmospheric noise. However, the SID concerned occurred between 0430 and 0500 U.T. on 16 August 1958. Atmospheric noise records for this day indicate no local sources, while sferics bearing observations covering the period of the SID show the activity to be to the east and to the southwest of Japan [IGY Data, Japan, 1959]. It seems plausible to associate these directions respectively with the main storm centers in the Americas and in SE Asia. The activity in the Americas will be dying with the advent of local night; that in SE Asia will be rapidly increasing the time being adjacent to local noon. In atmospheric noise measurements the nearest storms are the dominant factors [Horner, 1960]; thus the SE Asia center is likely to be more important than that in the Americas. Furthermore, it is the closer parts of the SE Asia center that will be most vital; this effect is enhanced by the influence of time since maximum thundery activity occurs in the local afternoon, and it is generally in the nearer parts of the SE Asia center to Japan, that, at the time of the SID, the local maximum is most closely approached. Considering all factors, it seems reasonable to identify the principal sources of the atmospherics as being within the nearest landmasses to the southeast of Japan, and to take $d$ as being 2,000 $\mathrm{km}$. Using $h_{N} / h_{S}=1.08$, the values of $\left(\alpha_{S}-\alpha_{N}\right)$ were calculated as before and are represented by curve (b) on figure 2.

Obayashi [1960] has given two further examples of enhancements of atmospheric noise which are suitable for analysis. These are for 29 March 1959 and 16 June 1959. The distance of the sources of noise for the first instance is estimated at 2,000 $\mathrm{km}$, and Obayashi states that the storms were rather closer for the second enhancement; $d=1,500$ $\mathrm{km}$ would seem to be a reasonable mean estimate in this case. Using $h_{N} / h_{S}=1.08$ as before, the results obtained are shown in curves (c) and (d) on figure 2.

The agreement between the four curves in figure 2 , for the range of 8 to $35 \mathrm{kc} / \mathrm{s}$, is surprisingly good considering the speculative nature of the estimates for $d$ in particular. Above $35 \mathrm{kc} / \mathrm{s}$ and below 8 $\mathrm{kc} / \mathrm{s}$, the signal strengths involved are low, and thus the results are more prone to errors. This effect may account for the spread between 35 and $50 \mathrm{kc} / \mathrm{s}$, but seems quite inadequate to explain the discrepancies below $8 \mathrm{kc} / \mathrm{s}$. Here the divergence between Gardner's and Obayashi's work is very considerable. The estimate for $d$ is probably better for Gardner's observations than for Obayashi's; however, this factor is insufficient to enable the disagreements to be reconciled, and further experimental work is obviously desirable.

An intriguing feature of three of the curves on figure 2 is a maximum value and then a drop in $\left(\alpha_{S}-\alpha_{N}\right)$ as frequency decreases below $10 \mathrm{kc} / \mathrm{s}$. If this trend is continued it is probable that at some lower frequency, possibly in the extremely low frequency (ELF) range $(<3 \mathrm{kc} / \mathrm{s}),\left(\alpha_{S}-\alpha_{N}\right)$ will become negative as it is at frequencies above about $15 \mathrm{kc} / \mathrm{s}$.
It might be predicted that a SID will produce an enhancement of atmospheric noise above $15 \mathrm{kc} / \mathrm{s}$; a decrease at intermediate frequencies; and an enhancement again in the ELF band. This kind of predicted behavior, which would have analogies with the usual variation of $\alpha_{N}$ with frequency, could readily be verified by an extension of experimental observations to lower frequencies.

\section{Discussion}

Figure 3 is an attempt to summarize the preceding information. Curve (a) is an average representation for $\alpha_{N}$ derived from the two graphs of figure 1 . Curve (b) is a composite graph for $\alpha_{S}$; it was obtained by considering the four curves of figure 2, deducing weighted mean values for $\left(\alpha_{N}-\alpha_{S}\right)$, and using these, in conjunction with curve (a) of figure 3 to derive curve (b).

It is apparent from figure 3 that the frequency of $27 \mathrm{kc} / \mathrm{s}$ which has been established for many years [Bureau, 1937] as an indicator of enhancements in atmospherics is a good choice, since the minimum of $\alpha_{S}$ is close to $27 \mathrm{kc} / \mathrm{s}$ and the change between $\alpha_{N}$ and $\alpha_{S}$ is considerable. It is equally true that the differences between $\alpha_{N}$ and $\alpha_{S}$ are most marked for the lower frequencies, and the decrease in atmospherics over that frequency range is potentially a more sensitive indicator of the effects of a SID than is the enhancement at frequencies exceeding some $15 \mathrm{kc} / \mathrm{s}$. This is particularly so if the normal storm centers are sufficiently close to the observing station for adequate daytime signal strength to be always present. An interesting feature of figure 3 is that the band (15 to 20 $\mathrm{kc} / \mathrm{s}$ ) occupied by the most important VLF transmitters is little affected in attenuation by the onset of SID conditions.

The normal atmospherics receiver on $27 \mathrm{kc} / \mathrm{s}$ which is usually intended as an indicator of the sudden enhancements associated with a SID, can also give increased signals produced by source effects and not by the propagation changes due to the SID. Three main causes may be assigned to such increases, namely, an augmentation of the

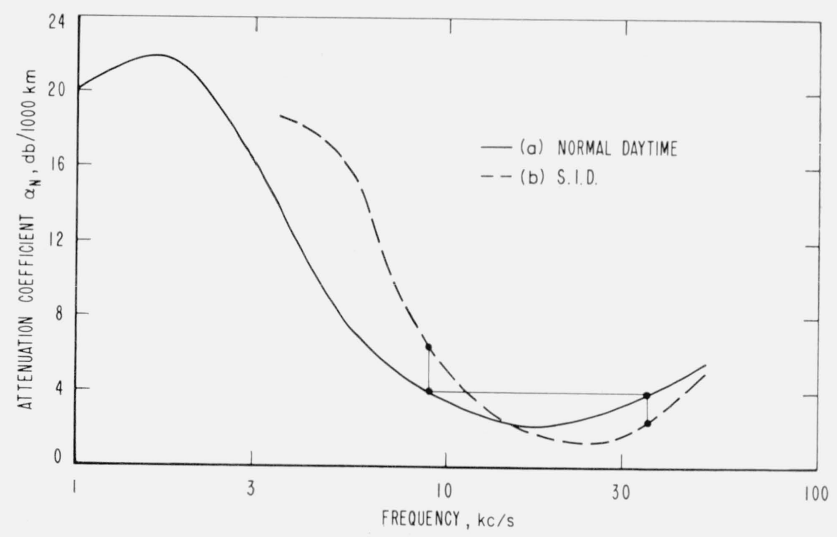

Figure 3. Composite values for attenuation coefficients under normal daytime and under SID conditions. 
activity in an existing storm center; the development of a new storm center; and the movement of existing activity closer to the observing station. It seems possible, upon the basis of figure 3, to produce a reciprocal instrument which would not respond to the above source effects, but would only register a change in propagation conditions such as that produced by a SID. The principle would be to select two frequencies $f_{1}$ and $f_{2}$, one on each side of the normal minimum for $\alpha_{N}$ at around $18 \mathrm{kc} / \mathrm{s}$, and for which the values of $\alpha_{N}$ are equal; as an example figure 3 indicates that 9 and $35 \mathrm{kc} / \mathrm{s}$ could be chosen for $f_{1}$ and $f_{2}$. Then if $E\left(f_{1}\right)$ and $E\left(f_{2}\right)$ are the measured fields at $f_{1}$ and $f_{2}$, the ratio $E\left(f_{1}\right) / E\left(f_{2}\right)$ should remain effectively constant irrespective of alterations in the strengths of the sources of atmospheric noise, or of changes in the distances of the sources, provided that these are not such as greatly to modify the validity of the basic onemode assumption; this implies distances always greater than some $1,000 \mathrm{~km}$. However if propagation conditions are affected as in a SID, the ratio $E\left(f_{1}\right) / E\left(f_{2}\right)$ will alter very considerably because of the changes in attenuation coefficient from $\alpha_{N}$ to $\alpha_{S}$. The effect will be especially marked being reciprocal at the two frequencies, as is shown in figure 3 for the selected frequencies of 9 and 35 $\mathrm{kc} / \mathrm{s}$. Construction of a simple recorder presenting the ratio $E\left(f_{1}\right) / E\left(f_{2}\right)$ directly offers no great difficulty.

It is outside the scope of this paper to examine the interpretation of the $\alpha_{S}$ values of figure 3 upon the waveguide mode theory. However, there is no reason to believe that the values are incompatible with the theory, indeed the opposite is rather the case and the results are further evidence - although this is hardly necessary - of the general validity of the mode interpretation. For instance, it can readily be deduced from the work of Wait [1957] that a reduction in $h$ from 70 to $60 \mathrm{~km}$, without any considerable associated change in ionospheric conductivity, produces two graphs very similar to those depicted on figure 3 . In each case, there is a reciprocal relative displacement of the two curves, pivoting around a common point.

\section{References}

Bracewell, R. N., and T. W. Straker, The study of solar flares by very long radio waves, Monthly Notices of Royal Astronomical Society, 109, 28-45 (1949).

Bureau, R., Abnormalities of the ionosphere and bright solar eruptions, Nature 139, 110-111 (1937).

Chapman, F. W., and R. C. Macario, Propagation of audio frequency radio waves, Nature 17\%, 930-933 (1956).

Friedman, H., Rocket observations of the ionosphere, Proc. IRE 4\%, 272-280 (1959).

Gardner, F. F., The use of atmospherics to study the propagation of very long radio waves, Phil. Mag. 41, 1259-1269 (1950).

Horner, F., Properties of natural noise, Survey Paper before Commission IV, URSI XIIIth General Assembly, London, 1960.

IGY Data on Atmospheries, Whistlers, and Solar Radio Emissions, National Committee for the IGY, Japan, Vol. III for July-Dec. 1958, 1959.

Obayashi, T., Measured frequency spectra of very low frequency atmospherics, J. Research NBS 64D, 41-48 (1960).

Obayashi, T., S. Fujii, and T. Kidokoro, An experimental proof of the mode theory of VLF ionospheric propagation, J. Geomagnetism and Geoelectricity, 10, 47-55 (1959).

Pierce, E. T., and D. W. Swift, Study of spontaneous ionospheric disturbances, Research and Advanced Development Division, AVCO Corporation, Final Report for Geophysics Research Directorate, contract No. AF29(601)-1985 (1960).

Wait, J. R., The attenuation vs frequency characteristics of VLF radio waves, Proc. IRE 45, 768-771 (1957).

Wait, J. R., A study of VLF field strength data: both old and new, Geofisica pura e applicata 41, 73-85, (1958).

Watt, A. D., and R. W. Plush, Power requirements and choice of an optimum frequency for a world-wide standardfrequency broadcasting station, J. Reaserch NBS, 63D, 35-44 (1959).

(Paper 65D6-158) 